\title{
Viral Disease Goes Viral: Characterizing How Cancer Patients Use Internet Resources for COVID-19 Information
}

\author{
Ruijia Jin ${ }^{1} \cdot$ Howard J. Lim ${ }^{2,3} \cdot$ Sarah Hamilton ${ }^{4,5} \cdot$ Paris-Ann Ingledew ${ }^{4,5}$ (I)
}

Accepted: 7 January 2022 / Published online: 13 January 2022

(c) The Author(s) under exclusive licence to American Association for Cancer Education 2022

\begin{abstract}
This study aims to evaluate the usage of Internet resources for COVID-19 information among cancer patients. Specifically, to understand where patients are seeking information, what topics are most frequently searched, and how physicians and web developers can improve clinical conversations and digital resources, respectively, to support cancer patients' needs. From May to June 2021, cancer patients who were attending follow-up at a tertiary cancer center completed a survey consisting of 28 closed and open-ended questions. Quantitative results were evaluated using descriptive statistics and qualitative responses were evaluated using a grounded-theory approach. Fifty-seven surveys were distributed, and fifty-two surveys were received (91\% response rate). The majority of respondents (96\%) were Internet users. Seventy percent used the Internet as a source of information about COVID-19 and cancer personally, with another 15\% reporting that friends and family accessed online information on their behalf. The vast majority used Google as their choice of search engine, with COVID-19 rates and vaccine information being the most frequently searched topics. Three quarters (74\%) considered Internet information easy to understand, and 90\% stated that the Internet increased their understanding of COVID-19 and cancer. Only 15\% of patients had been recommended online resource(s) by a physician, yet $100 \%$ of those patients found the physician-recommended sites useful. Most cancer patients use the Internet to search for COVID-19 information. Healthcare professionals (HCPs) should help guide patients towards credible online sources and address knowledge gaps to improve physician-patient communication and support educational needs.
\end{abstract}

Keywords COVID-19 $\cdot$ Cancer $\cdot$ Internet $\cdot$ Online resources $\cdot$ Patient education $\cdot$ Web resources

Paris-Ann Ingledew

pingledew@bccancer.bc.ca

1 Faculty of Medicine, MD Undergraduate Program, University of British Columbia, 317-2194 Health Sciences Mall, Vancouver V6T 1Z3, Canada

2 Medical Oncology, Vancouver Centre, BC Cancer Agency, 600 West 10th Avenue, Vancouver V5Z 4E6, Canada

3 Division of Medicine, Faculty of Medicine, University of British Columbia, 2775 Laurel Street, Vancouver V5Z 1M9, Canada

4 Radiation Oncology, Vancouver Centre, BC Cancer Agency, 600 West 10th Avenue, Vancouver V5Z 4E6, Canada

5 Division of Surgery, Faculty of Medicine, University of British Columbia, 2775 Laurel Street, Vancouver V5Z 1M9, Canada

\section{Introduction}

COVID-19, a viral disease caused by SARS-CoV-2, has spread in an infectious sense both physically and virtually. With more than 1,400,000 total cases and 26,000 deaths in Canada since its induction in early 2020, it is a disease that has triggered widescale policies such as quarantine protocols, mask mandates, social distancing, and even lockdown, while also garnering intense media attention [1]. Cancer patients, especially those with metastatic cancer (stage IV), have been shown to be more vulnerable to severe disease and hospitalization with COVID-19 infection [2].

The Internet has been an important tool throughout the COVID-19 pandemic. A recent Canadian study showed that $68 \%$ of Canadian adults reported increasing their Internet use during COVID-19 [3], and nearly all major Internet services saw increased traffic since early March 2020 [4]. Statistics Canada reported in 2018 that $91 \%$ of Canadians over 15 years of age used the Internet [5]. Recent studies 
have shown that cancer patients, specifically, frequent the Internet to search for information on their cancer and guide discussions with their HCPs [6-10]. A trend analysis in the USA showed that there was a $16 \%$ increase in internet usage in cancer survivors between 2011 and 2017 [11].

In the current literature, there is some information on COVID-19-related Internet search patterns, but little to no information specific to cancer patients. A study published in BMJ analyzed COVID-19 search patterns among people in the USA. Results showed that the most popular search terms were transmission, clinical signs and symptoms, and activity modification; in addition, many searches were about myths associated with the disease [12]. Another US study illustrated that Internet search data reflected news of local COVID-19 cases: people responded to the first COVID-19 report in their state by immediately seeking information related to COVID-19, especially coronavirus symptoms and hand sanitizer [13].

This survey study was done adjacently with an analysis on the quality of COVID-19 and cancer Internet resources which is in the process of manuscript submission. The analysis showed variable quality of COVID-19 and cancer websites, with many sites lacking markers for accountability such as author disclosure, citations, and recency of publication or updates.

Despite the prevalence of cancer patients' Internet usage and the variable quality of currently available online COVID-19 resources for cancer patients, little is known about how cancer patients use the Internet for COVID-19 information and how often they evaluate the quality of this information. The purpose of this survey study is to characterize how cancer patients use Internet resources to look for COVID-19 and cancer information, as well as the effect of online information on infection prevention measures, cancer appointments/tests, COVID-19 vaccine willingness, and clinical interactions with healthcare providers.

\section{Methods}

From May to June 2021, cancer patients receiving care from radiation oncologists and medical oncologists at a tertiary cancer center in Canada were invited to participate in this study. At this time, Canada was in the third wave of the pandemic, around 18 months after the start of COVID-19. Eligible participants were identified by searching a database of patients attending follow-up clinics. Patients were included if they had a diagnosis of cancer, were 19 years or older, were able to provide voluntary consent, and had adequate English language knowledge to understand the study and complete the survey with respect to their use of English websites. Patients were excluded if they did not meet all of these criteria. After the oncologist screened for eligibility, the medical student researcher followed up with eligible patients by phone or in-person to provide a thorough explanation of the consent form and study. Participants who wanted to participate were then given the choice of receiving a paper copy of the survey and consent form with a stamped, pre-addressed envelope or emailed an online link with a unique identifier. The online version was created using University of British Columbia's secure cloud-based platform provided by Qualtrics. Participation was voluntary and all responses were anonymized before analysis. This study was approved by the local cancer agency Research Ethics Board, certificate $\mathrm{H} 21-00,791$.

The survey consisted of 28 open- and closed-ended questions. To develop the survey, a review of the literature was done regarding patient Internet use with a focus on cancer patients [14] and best practice strategies for medical education surveys $[15,16]$ were incorporated. To date, this survey has been used and iteratively reviewed and refined for a process of validation in more than a dozen unique tumor sites by our research team in the past decade [6-10]. The survey included patient demographics (age, type of cancer, date of diagnosis), Internet usage patterns (frequency, device, use of Internet as a source for COVID19 and cancer information), search patterns (search engines and search terms used, social media usage, physician recommended sites), website selection and evaluation, the usefulness of the Internet as a resource for COVID-19 and cancer information (usefulness, ease of understanding, discrepancy or discussion with HCP), and the Internet's influence on thoughts and actions related to COVID-19 (prevention measures, attending cancer appointments during COVID, willingness to get vaccinated, anxiety and fear regarding COVID, treatment decisions and clinical relationship with $\mathrm{HCP}$, benefit of Internet compared to other resources).

Quantitative data was analyzed using descriptive statistics on Microsoft Excel. Qualitative data was analyzed using a grounded theory approach [17]. Two investigators (a radiation oncologist and a second-year medical student/ clinical research assistant) met a priori and discussed a coding structure using a narrative analysis method. The initial structure was grounded in prior studies done by the research group relating to patient factors in Internet use as well as themes in existing literature. The investigators then reviewed the narrative responses and open-ended questions then discussed and identified themes. Any coding discrepancy was resolved through iterative discussion. These themes were then used to categorize answers and exemplary quotes were noted. Due to the small amount of data, NVivo was not requested and Microsoft Excel was adequate for grouping and keeping track of themes. 


\section{Results}

The survey was offered to 57 patients who had expressed interest during the oncologist screening. Fifty-two people completed and returned the survey, which was a $91 \%$ response rate. Patient demographics are shown in Table 1.

\section{Internet Usage}

Ninety-six percent of participants $(n=50)$ reported using the Internet, with $6 \%(n=3)$ using it rarely $(<4$ times per month), $8 \%$ using it often (1-4 times per week), and $82 \%$ $(n=43)$ using it regularly (1-4 times per day). The majority of Internet users $(70 \%, n=35)$ personally used the Internet as a source of information about COVID-19 and cancer. Out of the 17 people who reported not using the Internet for this information, the majority $(71 \%, n=12)$ stated the reason was because they received sufficient information from news channels and did not feel the need to look for information online, $18 \%(n=3)$ stated they were satisfied by information

Table 1 Demographics of survey respondents

\begin{tabular}{llll}
\hline Variable $(n=52)$ & Number & Percentage (\%) & $\begin{array}{l}\text { People who are CC } \\
\text { Internet users* }(\%)\end{array}$ \\
\hline Age & & & \\
$19-39$ & 2 & 4 & 100 \\
$40-59$ & 15 & 29 & 93 \\
$60-79$ & 32 & 62 & 72 \\
& & & d \\
80-99 & 3 & 6 & 0 \\
Type of cancer & & & \\
$\quad$ diagnosis & & & 75 \\
Head and neck & 4 & 8 & 88 \\
Breast & 8 & 15 & 75 \\
Lung & 4 & 8 & 76 \\
Gastrointestinal & 29 & 56 & 50 \\
Gynecological & 4 & 8 & 50 \\
Prostate & 2 & 4 & 100 \\
Sarcoma & 1 & 2 & \\
Palliative or curative & & & 70 \\
Palliative & 30 & 58 & 82 \\
Curative & 22 & 42 & 50 \\
Year of diagnosis & & & 100 \\
2018-2021 & 31 & 60 & 17 \\
2014-2017 & 9 & 8 & \\
2009-2013 & 8 & 15 & \\
<2009 & 4 & 86 \\
\hline
\end{tabular}

* "CC Internet users" are either (a) a cancer patient who used the Internet to look for COVID-19 and cancer information or (b) a family member or friend of a cancer patient who used the Internet to look for COVID-19 and cancer information on behalf of the patient provided by HCPs, and $12 \%(n=3)$ stated they were confused or overwhelmed by the Internet. Also out of these 17,8 respondents reported that family or friends accessed the Internet on their behalf. Out of those 8 surveys, 4 of them had a family member or friend available who filled out the rest of the survey. In total, $39(35+4)$ participants were classified as available "cancer-COVID Internet users" (CC Internet users): either a cancer patient or a family member/ friend who used the Internet as a source of information about COVID-19 and cancer.

\section{Internet Search Patterns}

Ninety-two percent $(n=36)$ of CC Internet users used a search engine to search for COVID-19 and cancer information, with the vast majority $(97 \%, n=35)$ using Google as their choice search engine. One respondent reported also using a news search engine and another used solely DuckDuckGo. The most commonly searched term was COVID-19 or COVID $(n=27)$. Other commonly searched terms were vaccine(s) $(n=14)$ and cancer $(n=11)$. One third $(n=13)$ of CC Internet users reported accessing information on social media or social networking sites, with Facebook $(46 \%, n=6)$ and YouTube $(46 \%, n=6)$ being the most popular sites.

To select which sites to view for information, the majority of CC Internet users looked at specific pages from reputable sources $(67 \%, n=26)$. Others followed top hits from search engine results $(46 \%, n=18)$, recommendations from friends or family $(36 \%, n=14)$, or recommendations from health care providers $(15 \%, n=6)$. Users typically reviewed a total of $1-5$ websites $(56 \%, n=22)$, while $26 \%(n=10)$ reviewed $6-10,13 \%(n=5)$ reviewed $11-15$, and $5 \%(n=2)$ reviewed more than 20 sites.

A detailed breakdown of COVID-19 and cancer topics that were searched most frequently is shown in Table 2. Participants most commonly sought information on vaccines $(90 \%, n=35)$ and infection rates $(77 \%, n=30)$, followed by risk factors $(64 \%, n=25)$. Four narrative responses also mentioned searching for information on COVID-19 hotspot and outbreak areas.

\section{Websites Accessed}

The survey included an open-ended question for respondents to list specific websites they recalled visiting. Out of 39 CC Internet users, 35 listed specific sites. Fifty-one percent of sites were government or institutional/academic websites (i.e., provincial government site, Center for Disease Control), 19\% were news sites (i.e., CNN, Global News), $18 \%$ were non-profit organizations (i.e., Mayo Clinic, Canadian Cancer Society), and $11 \%$ were commercial sites (i.e., WebMD, Healthline). One respondent reported reading a scientific journal. Out of 35 respondents, 32 accessed 
Table 2 Topics most commonly searched for in patients who have sought online information on COVID-19 and cancer, ranked from most popular to least popular

\begin{tabular}{ll}
\hline Topic & $\begin{array}{l}\text { Patients who have reported seeking } \\
\text { information on it, } n \text { (\% of CC Internet } \\
\text { users) }\end{array}$ \\
\hline COVID-19 vaccine information & $35(90 \%)$ \\
Rates of COVID-19 & $30(77 \%)$ \\
Risk factors for COVID-19 & $25(64 \%)$ \\
Symptoms of COVID-19 & $23(59 \%)$ \\
Special considerations for cancer patients during the COVID-19 & $22(56 \%)$ \\
Pandemic & $21(54 \%)$ \\
prevention of COVID-19 & $15(39 \%)$ \\
Definition of COVID-19 & $11(28 \%)$ \\
Treatment of COVID-19 & $10(26 \%)$ \\
Detection/workup of COVID-19 & $6(15 \%)$ \\
Prognosis of COVID-19 & $3(8 \%)$ \\
Discussion boards/forums & $2(5 \%)$ \\
Education support (workshops, modules) &
\end{tabular}

* "CC Internet users" are either (a) a cancer patient who used the Internet to look for COVID-19 and cancer information or (b) a family member or friend of a cancer patient who used the Internet to look for COVID-19 and cancer information on behalf of the patient
Canadian websites. The most popular sites included provincial government sites $(n=11)$, the Center for Disease Control $(n=8)$, Mayo Clinic $(n=7)$, and Canadian Cancer Society $(n=5)$.

\section{Evaluation of Websites}

Most participants $(74 \%, n=29)$ found the Internet information easy to understand, while $21 \%(n=8)$ found the information somewhat hard to understand, and 5\% $(n=2)$ found the information difficult to understand. The majority of respondents $(59 \%, n=23)$ reported evaluating the quality of online information all the time, $31 \%(n=12)$ some of the time, and $10 \%(n=4)$ never. The most common method for evaluation was using credible sources such as academic or government websites $(85 \%, n=33)$. Other methods included examining references $(49 \%, n=19)$, comparing with other websites $(44 \%, n=17)$, and referring to author credentials $(41 \%, n=16)$. Eighteen percent $(n=7)$ of participants discussed online information with their healthcare provider. A more detailed look at website evaluation methods arranged by patient age is provided in Table 3.

Fifteen percent $(n=6)$ of participants reported being recommended online resources by a physician or other HCP. Sixty-seven percent of these respondents $(n=4)$ were recommended a provincial cancer agency website, $50 \%$ $(n=3)$ the Canadian Cancer Society site, and 17\% $(n=1)$ UpToDate. Others had been recommended getvaccinated. ca $(n=1)$ and a provincial government website $(n=1)$. All users $(100 \%, n=6)$ found the website recommendations useful. One patient who received recommendations commented "All websites... clarified a doctor consultation or prepared me for questions to ask prior to consultation." Another reported "Some websites provided general and background information. Others [were] more medical in scope and function."
Table 3 Rates at which different age groups use specific methods to evaluate information found online $(n=39)$
Information evaluation method Frequency of method use by patient age, $n$ (\% of CC Internet users)

\begin{tabular}{llll} 
& $\mathbf{1 9 - 3 9}(n=2)$ & $\mathbf{4 0 - 5 9}(n=14)$ & $\mathbf{6 0 - 7 9}(n=23)$ \\
\hline Discussing with HCP & $0(0 \%)$ & $3(21 \%)$ & $4(17 \%)$ \\
Examining cited references & $2(100 \%)$ & $4(29 \%)$ & $13(57 \%)$ \\
Checking website authors' credentials & $2(100 \%)$ & $3(21 \%)$ & $10(43 \%)$ \\
Using credible sources & $2(100 \%)$ & $11(79 \%)$ & $20(87 \%)$ \\
Comparing several websites & $2(100 \%)$ & $5(36 \%)$ & $10(43 \%)$ \\
\hline
\end{tabular}

* "CC Internet users" are either (a) a cancer patient who used the Internet to look for COVID-19 and cancer information or (b) a family member or friend of a cancer patient who used the Internet to look for COVID-19 and cancer information on behalf of the patient 
Six users (15\%) noted that some information they found online conflicted with information provided by a healthcare professional. Comments included "[there was] mixed information from health authorities vs. NACI about mixing and matching vaccines" and "News online conflicted with my family doctor. For the Moderna vaccine, my family doctor said 12 weeks is the maximum amount of time [between doses] for the booster to be effective while the news said something different." Most participants $(69 \%, n=27)$ had not spoken with their healthcare provider about the knowledge they gained online. Narrative comments explained that this was mostly because the information online was in congruence with what their provider told them, and they had no further questions. Two respondents said COVID was not a topic of discussion during health care consultations and one respondent said they only go to trusted sites. Most of those who did speak with an HCP about online information reported asking about whether or not they should get the COVID-19 vaccine in the context of their cancer diagnosis and treatment. Some wanted to discuss, clarify, and verify the medical information they accessed online.

\section{Impact of Online Resources}

Most CC Internet users $(90 \%, n=35)$ found the Internet to be a useful source of information on COVID-19 and cancer. Some narrative comments talked about themes of efficiency, detail of information, and ease of access: "efficient to find information [online] instead of making appointments with doctors [who have] waiting periods," "[the Internet] has defined information sources [which] provided awareness and tools to navigate through COVID-19, allowing me to protect myself with science-based protocols since I have cancer vulnerabilities," "instant and easy access to info." Those who did not find the Internet useful reported conflicting information, politicization, and lack of sufficient information: "contrasting points of view, conflicting information, don't trust it," "too politicized, cannot tell what is reliable," "my type of cancer is not very common, and it has been studied just for a few years."

Ninety percent $(n=35)$ of users found that online information increased their overall understanding of COVID-19 and cancer. The Internet was somewhat influential as well - a breakdown of the Internet's influence on COVID-19 prevention measures, willingness to attend cancer appointments/ tests, and willingness to get the vaccine is illustrated in Fig. 1. Many respondents found the Internet somewhat influential for COVID-19 prevention, but not that influential when it came to attending in-person cancer appointments and tests or getting the vaccine. Anxiety or fear towards COVID-19 was not affected by the Internet for $67 \%(n=26)$ of users, worsened by the Internet for $28 \%(n=11)$, and reduced by the Internet for $5 \%(n=2)$. Internet information did not affect clinical

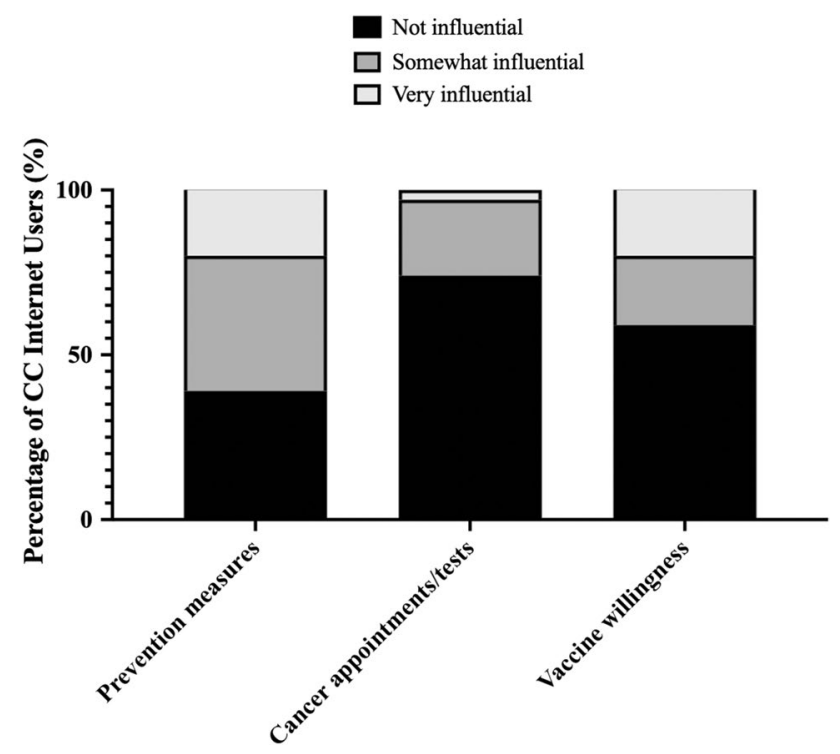

Fig. 1 Influence of COVID-19 and cancer online resources on COVID-19-related thoughts/actions

consultations and decision-making for the majority of users $(80 \%, n=31)$, helped in decision-making for $18 \%(n=7)$, and made decision-making more difficult for $3 \%(n=1)$.

Forty-four percent $(n=17)$ of CC Internet users found volume and detail of information to be the most important factor that the Internet offers compared to other COVID-19 and cancer resources, $31 \%(n=12)$ found ease of access to be of greatest importance, and 13\% $(n=5)$ opted for discussion of different perspectives and options. Narrative responses included convenience for the visually impaired $(n=1)$, indepth information $(n=1)$, less hassle $(n=1)$, and ability to keep knowledge current $(n=1)$.

An open-ended question on the survey asked if there were any topics related to COVID-19 and cancer that respondents felt the Internet did not cover. Out of 39 CC Internet users, 9 wrote a narrative response to this question. The two topics most users mentioned were COVID-19 risk in cancer and vaccine information especially in the context of cancer.

Twenty-three out of 39 users filled out the open-ended "additional comments" box, which was the last question of the survey. Eight comments were regarding vaccines, either asking a question about vaccines or requesting more information on them. Others talked about COVID-19 risk in cancer, news information, and the variability of online information.

\section{Discussion}

This study characterizes how cancer patients use the Internet for COVID-19 and cancer information and how it influences their understanding, clinical consultations, and actions. At 
the time of the study, during Canada's third wave, the vaccine had been approved and released for 3-4 months, but most Canadians had not yet been fully vaccinated. The timing of the survey should be considered upon interpretation of the findings when detailing what information people were seeking. For example, people may have been seeking information on not only COVID-19 but also vaccines and "long COVID" sequelae, which was likely not the case a year earlier. Nearly all cancer patients surveyed used the Internet and $83 \%$ were CC Internet users: either patients who accessed COVID-19 and cancer information online themselves or had family and friends who accessed information online on their behalf. Not surprisingly, the youngest age bracket had the highest percentage of CC Internet users, and as we moved up in age bracket, the percentage decreased. These numbers are similar to previous studies looking at cancer patients accessing online information for their specific cancer types [6-10]. In patients who did not use the Internet for COVID-19 and cancer information, the most common reason stated was that they received sufficient information from news outlets. Some also reported satisfaction with information provided by healthcare providers while others reported feelings of being overwhelmed by the web.

It is important to learn about how cancer patients sift through and select websites to view when looking for COVID-19 and cancer information. Many of our findings are consistent with previous studies done looking at general cancer information seeking patterns online by cancer patients. Congruent with past studies on skin, gynecologic, testicular cancer, breast, and thyroid cancer patients [6-10], nearly all COVID-19 and cancer searches began with a Google search. Most CC Internet users selected websites based on pages from specific reputable sources such as government sites or academic medical institutions. Nearly half, however, used top hits from search engines to choose websites to view. This is not ideal as the top hits returned by Google's ranking algorithm may not produce the highest quality medical information [18]. In addition, Google searches may be biased by previous searches affecting the algorithm. This is especially concerning as users in this study typically only reviewed 1-5 websites, which is consistent with the typical consumer [19]. In addition, some patients chose sites recommended by family and friends while a smaller number chose sites based on recommendations from doctors or other HCPs. A little more than half of participants reported consistently evaluating the quality of online information they view. The most popular method to do this was accessing credible sources, followed by examining references, comparing different sites, and referring to author credentials. Interestingly, as shown in Table 2, the 60 to 79-year-olds seemed to evaluate online information more readily than the 40- to 59-year-old age bracket, winning out in percentage for almost all methods (examining cited references, checking website authors' credentials, using credible sources, comparing data from several websites) other than discussion with HCP. Previous studies have shown that older age is inversely correlated with Internet savviness [20], so our results are surprising as we would expect a younger generation to be more computer-literate and engage more with website evaluation. In addition, a similar study on thyroid cancer patient health information-seeking patterns showed contrasting results to our study - their oldest age bracket had the lowest percentage use of information evaluation methods for most methods. Our hypothesis to explain our findings was that older patients are more skeptical or worried about COVID-19 and cancer information on the Internet and may be doing more research to ensure reliable information. Conversely, younger patients may be more confident in the information they receive on the Internet and less focused on detailed evaluation of the resources.

The quality of web resources can be highly variable. Previous studies have found variable quality of COVID-19 prevention and treatment information [21, 22]; our recent website analysis COVID-19 and cancer resources (completed adjacently with this survey study) found that many accountability markers such as authorship, citations, and recency were lacking for many websites. This highlights the essential role of healthcare providers to help patients navigate online resources and find reliable information.

Most patients found the online resources understandable, but $25 \%$ did find the information at least somewhat hard to understand, reasonably consistent with previous studies [6-10]. Difficulty in understanding may be due to excessive use of medical jargon, consistent with our website analysis which found that all COVID-19 and cancer websites were written above recommended reading level for public health information. Ways to improve readability and ease of understanding of health information include limiting sentences to 8-10 words and using simple words instead of complicated medical terminology [23]. In addition, illustrations included in health education text have been shown to make literature more accessible and appealing to individuals at all levels of reading ability [24].

When asked to list search terms, the most common ones written were COVID-19 or COVID, vaccine(s), and cancer. For specific topics, vaccine information was most popular, followed by COVID-19 rates and risk factors. Referencing our website analysis, COVID-19 vaccine information was the topic most likely to be inaccurate when covered by websites, with $33 \%$ of sites providing inaccurate content. In addition, the most commonly accessed websites according to our survey were provincial government sites, the Center for Disease Control, and Mayo Clinic. According to our website analysis, $67 \%$ of government-owned sites were found to be inaccurate due to out-of-date vaccine information. This shows a strong need to improve the frequency of updates for 
health education sites, especially those provided by government and academic institutions to meet patients' needs.

Only a small fraction of CC Internet users reported that a physician recommended any COVID-19 and cancer websites to them, with the provincial cancer agency and the Canadian Cancer Society being the most commonly mentioned sites. One hundred percent of those who were recommended sites, however, stated that the recommended websites were useful. Comments praised about how these sites "helped [them] navigate [their] therapy processes, clarify[ing] a doctor consultation or prepar[ing] [them] for questions to ask prior to consultations." This clearly shows that patients and their family/friends appreciate when HCPs guide them to find reliable online sources, and this is a skill that more physicians should incorporate into future clinical interactions.

Less than a third of participants had ever spoken with a healthcare professional about COVID-19 and cancer information that they found on the Internet, while 15\% reported that Internet information had conflicted with information provided by a healthcare professional. Narrative explanations reported "mixed information... about mixing and matching vaccines" and confusion about maximum effective intervals between doses. These comments further emphasize the importance of physicians starting conversations about COVID-19 with cancer patients to mutually share resources and address potential misinformation. As one participant addressed with a narrative comment, any miscommunication also highlights that government agencies such as the National Advisory Committee on Immunization (NACI) should consider the messages they send out as it may conflict with messaging from HCPs; ideally, there need to be methods in place to ensure consistent messaging. The most common reason for speaking with HCPs about COVID-19 and cancer was - not surprisingly since this topic was also most commonly searched - discussion about efficacy and safety of COVID-19 vaccines in the context of cancer diagnosis and treatment.

About a third of CC Internet users reported using social media or social networking sites to access COVID-19 and cancer information, with Facebook and YouTube as top choices. Similar to Google searches, results from social media searches are likely to be affiliated with significant bias based on previous searches. Currently, there are few comprehensive studies about the quality of cancer information on social media platforms [25]. COVID-19 information on social media has been shown to be somewhat problematic as well, with a recent study presenting evidence that people often share false claims about COVID-19 when not directly asked about accuracy [26]. This is important to explore in further research as social media is expanding as a platform for seeking and sharing health-related information.

Nearly $90 \%$ of CC Internet users stated that the Internet was a useful source of information on COVID-19 and cancer, increasing their overall understanding on the topic. This number is quite consistent with previous studies, though there is a variation depending on the type of cancer. Some patients such as those with gynecologic cancers and melanomas found the Internet less useful for understanding their conditions than our survey participants while others, such as breast cancer, testicular cancer, and thyroid cancer patients found online information more useful in their health information exploration [6-10].

Despite the usefulness of the Internet, its influence was varied. Our survey asked participants to rate the Internet's influence on COVID-19 prevention measures, attitude towards attending in-person cancer appointments and tests, willingness to get the COVID-19 vaccine, and decisionmaking during consultations with physicians. It was most influential for prevention measures and least influential for decision-making in physician consultations. Previous studies have also found that while the Internet was a common information resource, physicians were considered far more trustworthy [27]. Online information influenced vaccine willingness for $41 \%$ of participants; this is important to note since in previous studies, it has been shown that getting information from the Internet or relatives increased the rate of vaccine hesitancy when compared to getting information from HCPs alone [28]. Additional narrative comments revealed major themes: many wished for more comprehensive online information on COVID-19 risk in cancer patients and vaccine information in the context of cancer. One participant, for example, commented "to what degree do cancer therapies dilute or threaten an individual's immune system and vulnerability to the variants?" Other general comments addressed the variable quality of online information and news resource access for COVID19 information. In addition, almost a third of CC Internet users felt that online information worsened their anxiety or fear towards COVID-19, which is a significant concern. This speaks to the responsibility of HCPs to ask patients about what online resources they have accessed and reassure them by answering questions and discussing potential misconceptions.

There are some limitations to address for this study. The sample size was small, and patients were recruited from one geographical site in Canada. Thus, the findings may not be generalizable to all cancer patients. As with all voluntary surveys, this study likely suffered from selection bias, as those who do not use the Internet or do not have strong opinions on the topic may have been less likely to participate in the survey. Recall bias was also possible, and some patients may not have wanted to disclose their sources of information despite the anonymous nature of the study. Another limitation is that our study included a large number of gastrointestinal cancer patients; in the future, more patients of other cancer sites could be explored. 


\section{Conclusion}

This is to our knowledge the first study to date exploring Internet usage patterns regarding COVID-19 in cancer patients. Our study makes a relevant contribution to the literature of health information seeking among cancer patients in the unique context of COVID-19. It has shown that most cancer patients are regular Internet users and search for COVID-19 information online often in the context of their cancer diagnosis, with particular emphasis on vaccine information and COVID-19 infection rates. Patients generally understand the information but may not always evaluate the quality of online resources. Most healthcare professionals currently do not take the time to recommend reliable online resources about COVID-19 for cancer patients, and it is a topic that is rarely brought up by patients themselves even after accessing information online. Healthcare providers should ask patients about the online resources they access and offer guidance on selecting credible sources while filling in knowledge gaps where online information may not be available. Educational websites on COVID-19 and cancer can use the results of this study to tailor content towards the informational needs of cancer patients.

Availability of Data and Material All websites are publically accessible. Data is contained within this article or supplementary material presented. The survey tool is available upon request from the corresponding author, but it is not publicly available due to its proprietary nature.

Code Availability All figures for data were generated using GraphPad Prism 9.1.9 for Mac, GraphPad Software, San Diego CA, USA, www. graphpad.com.

\section{Declarations}

Ethics Approval Ethical review and approval were obtained via the local cancer agency review board, H21-00791.

Consent to Participate Participants were provided information on the study and told that completion of the survey, either online or the physical copy, would indicate voluntary participation.

Consent for Publication Participants were informed that results of the study would be analyzed and potentially published.

Conflict of Interest The authors declare no competing interests.

\section{References}

1. Canada PHAof. Epidemiological summary of COVID-19 cases in Canada. Canada.ca. 2021. Available online: https://health-infob ase.canada.ca/covid-19/epidemiological-summary-covid-19cases.html (accessed 24 June 2021).
2. Dai M. et al. Patients with cancer appear more vulnerable to SARS-CoV-2: a multicenter study during the COVID-19 outbreak. Cancer Discov. 2020, Volume 10. doi: https://doi.org/10. 1158/2159-8290.CD-20-0422

3. Zajacova A, Jehn A, Stackhouse M et al (2020) Changes in health behaviours during early COVID-19 and sociodemographic disparities: a cross-sectional analysis. Can J Public Health 111:953-962. https://doi.org/10.17269/ s41997-020-00434-y

4. Koeze E, Popper N. The virus changed the way we internet. The New York Times. The New York Times. 2020. Available online: https://www.nytimes.com/interactive/2020/04/07/technology/ coronavirus-internet-use.html (accessed 24 June 2021)

5. Statistics Canada. Internet use at home, by Internet activity and urban or rural distribution. Available online: https://www150. statcan.gc.ca/t1/tbl1/en/tv.action?pid=2210006601 (accessed 24 June 2021).

6. Hamilton SN, Scali EP, Yu I, Gusnowski E, Ingledew P-A. Sifting through it all: characterizing melanoma patients' utilization of the Internet as an information source. J Cancer Educ. 2014. Volume 30. doi: https://doi.org/10.1007/s13187-014-0711-1

7. Mcleod J, Yu I, Ingledew P-A (2017) Peering into the deep: characterizing the Internet search patterns of patients with gynecologic cancers. J Cancer Educ 32:85-90. https://doi.org/ 10.1007/s13187-016-1002-9

8. Yeo S, Eigl B, Chan S, et al. Caught in the net: characterizing how testicular cancer patients use the internet as an information source. Can Urol Assoc J. 2021. Volume 15. https://doi.org/10. 5489/cuaj.6870

9. Nguyen SKA, Ingledew PA (2013) Tangled in the breast cancer web: an evaluation of the usage of web-based information resources by breast cancer patients. J Cancer Educ 28:662-668. https://doi.org/10.1007/s13187-013-0509-6

10. Chang K, Berthelet E, Grubbs E, Hamilton S, Karvat A, Tran E, Wu J, Ingledew PA (2020) Websites, Websites Everywhere: how thyroid cancer patients use the internet. J Cancer Educ 35:1177-1183. https://doi.org/10.1007/s13187-019-01576-5

11. Jiang S, Liu PL (2020) Digital divide and Internet health information seeking among cancer survivors: a trend analysis from 2011 to 2017. Psychooncology 29:61-67. https://doi.org/10. 1002/pon.5247

12. Shen T, Chen A, Bovonratwet P, Shen C, Su E. COVID-19related internet search patterns among people in the United States: exploratory analysis. J Med Internet Res. 2020. Volume 22. doi: https://doi.org/10.2196/22407

13. Bento A, et al. Evidence from internet search data shows information-seeking responses to news of local COVID-19 cases. Proceedings of the National Academy of Sciences. 2020. Volume 117. doi: https://doi.org/10.1073/pnas.2005335117

14. Adams E, Boulton M, Watson E (2009) The information needs of partners and family members of cancer patients: a systematic literature review. Patient Educ Couns 77:179-86

15. Fowler FJ (2014) Survey research methods. Sage Publication, London

16. Artino AR, La Rochelle JS, Dezee KJ, Gehlbach H (2014) Developing questionnaires for educational research: AMEE Guide No. 87. Med Teach 36:463-474. https://doi.org/10.3109/ 0142159X.2014.889814

17. Watling CJ, Lingard L (2012) Grounded theory in medical education research: AMEE Guide No. 70. Med Teach 34:850-861. https://doi.org/10.3109/0142159X.2012.704439

18. Pérez-López FR. An evaluation of the contents and quality of menopause information on the World Wide Web. Maturitas. 2004. Volume 49. pp. 276-82. doi: https://doi.org/10.1016/j. maturitas.2004.07.006. PMID: 15531123 
19. Google Organic CTR History. Advanced web ranking. Available online: https://www.advancedwebranking.com/ctrstudy/ (accessed 27 June 2021)

20. Chang J, Mcallister C, Mccaslin R. Correlates of, and barriers to, Internet use among older adults. Journal of Gerontological Social Work. 2014, pp. 66-85. doi:https://doi.org/10.1080/01634 372.2014.913754.

21. Jayasinghe R, Ranasinghe S, Jayarajah U, Seneviratne S (2020) Quality of online information for the general public on COVID19. Patient Educ Couns 103:2594-2597. https://doi.org/10.1016/j. pec.2020.08.001

22. Fan KS, Ghani SA, Machairas N, Lenti L, Fan KH, Richardson $\mathrm{D}$, et al. COVID-19 prevention and treatment information on the internet: a systematic analysis and quality assessment. BMJ Open. 2020, Volume 10. doi: https://doi.org/10.1136/bmjop en-2020-040487

23. Boztas N, Omur D, Ozbılgın S, Altuntas G, Piskin E, Ozkardesler S, Hanci V. Readability of internet-sourced patient education material related to "labour analgesia". Medicine (Baltimore). 2017. Volume 96. doi: https://doi.org/10.1097/MD.0000000000 008526

24. Michielutte Robert PhD, Bahnson Judy BA, Dignan Mark PhD, Schroeder Elissa PhD (1992) The use of illustrations and narrative text style to improve readability of a health education brochure.
J Cancer Educ 7:251-260. https://doi.org/10.1080/0885819920 9528176

25. Griffiths F, Cave J, Boardman F, Ren J, Pawlikowska T, Ball R, Clarke A, Cohen A (2012) Social networks-the future for health care delivery. Soc Sci Med 75:2233-41. https://doi.org/10.1016/j. socscimed.2012.08.023

26. Pennycook G, McPhetres J, Zhang Y, Lu JG, Rand DG (2020) Fighting COVID-19 Misinformation on social media: experimental evidence for a scalable accuracy-nudge intervention. Psychol Sci 31:770-780. https://doi.org/10.1177/0956797620939054

27. Inglehart RC, Taberna M, Pickard RK, Hoff M, Fakhry C, Ozer E, Katz M, Gillison ML (2016) HPV knowledge gaps and information seeking by oral cancer patients. Oral Oncol 63:23-29. https:// doi.org/10.1016/j.oraloncology.2016.10.021

28. Charron Charron Jalal, Gautier Arnaud, Jestin Christine (2020) Influence of information sources on vaccine hesitancy and practices. Médecine et Maladies Infectieuses 50:727-733. https://doi. org/10.1016/j.medmal.2020.01.010

Publisher's Note Springer Nature remains neutral with regard to jurisdictional claims in published maps and institutional affiliations. 